\title{
Improvement in cervical lordosis and sagittal alignment after vertebral body sliding osteotomy in patients with cervical spondylotic myelopathy and kyphosis
}

\author{
Dong-Ho Lee, MD, ${ }^{1}$ Choon Sung Lee, MD, ${ }^{1}$ Chang Ju Hwang, MD,1 Jae Hwan Cho, MD, ${ }^{1}$ \\ Jae-Woo Park, MD, ${ }^{1}$ and Kun-Bo Park, MD² \\ 'Department of Orthopedic Surgery, Asan Medical Center, University of Ulsan College of Medicine; and 2Division of Orthopedic \\ Surgery, Severance Children's Hospital, Yonsei University College of Medicine, Seoul, Korea
}

\begin{abstract}
OBJECTIVE Vertebral body sliding osteotomy (VBSO) is a safe, novel technique for anterior decompression in patients with multilevel cervical spondylotic myelopathy. Another advantage of VBSO may be the restoration of cervical lordosis through multilevel anterior cervical discectomy and fusion (ACDF) above and below the osteotomy level. This study aimed to evaluate the improvement and maintenance of cervical lordosis and sagittal alignment after VBSO.

METHODS A total of 65 patients were included; 34 patients had undergone VBSO, and 31 had undergone anterior cervical corpectomy and fusion (ACCF). Preoperative, postoperative, and final follow-up radiographs were used to evaluate the improvements in cervical lordosis and sagittal alignment after VBSO. C0-2 lordosis, C2-7 lordosis, segmental lordosis, C2-7 sagittal vertical axis (SVA), T1 slope, thoracic kyphosis, lumbar lordosis, sacral slope, pelvic tilt, and Japanese Orthopaedic Association scores were measured. Subgroup analysis was performed between 15 patients with 1-level VBSO and 19 patients with 2-level VBSO. Patients with 1-level VBSO were compared to patients who had undergone 1-level ACCF.

RESULTS C0 -2 lordosis $\left(41.3^{\circ} \pm 7.1^{\circ}\right)$, C2-7 lordosis $\left(7.1^{\circ} \pm 12.8^{\circ}\right)$, segmental lordosis $\left(3.1^{\circ} \pm 9.2^{\circ}\right)$, and C2 -7 SVA $(21.5 \pm 11.7 \mathrm{~mm})$ showed significant improvements at the final follow-up $\left(39.3^{\circ} \pm 7.2^{\circ}, 13^{\circ} \pm 9.9^{\circ}, 15.2^{\circ} \pm 8.5^{\circ}\right.$, and $18.4 \pm 7.9 \mathrm{~mm}$, respectively) after VBSO $(p=0.049, p<0.001, p<0.001$, and $p=0.038$, respectively). The postoperative segmental lordosis was significantly larger in 2-level VBSO $\left(18.8^{\circ} \pm 11.6^{\circ}\right)$ than 1 -level VBSO $\left(10.3^{\circ} \pm 5.5^{\circ}, p=\right.$ 0.014). The final segmental lordosis was larger in the 1-level VBSO $\left(12.5^{\circ} \pm 6.2^{\circ}\right)$ than the 1-level $\operatorname{ACCF}\left(7.2^{\circ} \pm 7.6^{\circ}, p=\right.$ $0.023)$. Segmental lordosis increased postoperatively $(p<0.001)$ and was maintained until the final follow-up $(p=0.062)$ after VBSO. However, the postoperatively improved segmental lordosis $(p<0.001)$ decreased at the final follow-up $(p=$ 0.045) after ACCF.
\end{abstract}

CONCLUSIONS Not only C2-7 lordosis and segmental lordosis, but also C0-2 lordosis and C2-7 SVA improved at the final follow-up after VBSO. VBSO improves segmental cervical lordosis markedly through multiple ACDFs above and below the VBSO level, and a preserved vertebral body may provide more structural support.

https://thejns.org/doi/abs/10.3171/2020.3.SPINE2089

KEYWORDS cervical myelopathy; cervical lordosis; sagittal vertical axis; anterior cervical corpectomy; vertebral body sliding osteotomy

A NTERIOR cervical corpectomy and fusion (ACCF) has been widely used for direct decompression as a treatment of multilevel myelopathy. ${ }^{1}$ However, high rates of implant dislodgement due to graft instability and dural tear during resection of the vertebral body have been reported. ${ }^{2-4}$ Patients with infection or tumor require a corpectomy, but if complete resection of the vertebral body is not essential, preserving a part of the vertebral body during anterior decompression would be better to minimize complications. For this purpose, a vertebral body sliding osteotomy (VBSO) procedure that allows for expansion of the spinal canal by anteriorly translating the vertebral body without complete removal has been introduced..$^{5,6}$

In addition to adequate decompression, restoration of

ABBREVIATIONS ACCF = anterior cervical corpectomy and fusion; ACDF = anterior cervical discectomy and fusion; ICC = intraclass correlation coefficient; JOA = Japanese Orthopaedic Association; NDI = Neck Disability Index; OPLL = ossification of the posterior longitudinal ligament; SVA = sagittal vertical axis; VAS-AP = visual analog scale for arm pain; VAS-NP = visual analog scale for neck pain; VBSO = vertebral body sliding osteotomy.

SUBMITTED January 20, 2020. ACCEPTED March 18, 2020.

INCLUDE WHEN CITING Published online May 22, 2020; DOI: 10.3171/2020.3.SPINE2089. 
cervical lordosis and improvement in sagittal alignment are important for better surgical outcomes, such as improved forward gazing, pain relief, and fewer adjacentlevel problems. ${ }^{7-11}$ While ACCF can achieve sufficient decompression, less improvement in cervical lordosis compared with multilevel anterior cervical discectomy and fusion (ACDF) has been reported., ${ }^{4,12}$ Two-level ACDF above and below the osteotomy is the first step of VBSO, and the preserved sliding vertebral body supports the trapezoidal-shaped interbody cage compared to ACCF. VBSO may facilitate achieving better postoperative cervical lordosis through multiple ACDFs and preservation of the vertebral body, i.e., if a 3-level ACDF is performed for a 2-level VBSO, cervical lordosis would increase more compared to a 2-level ACDF for a 1-level BVBSO.

Segmental cervical lordosis after cervical fusion affects C2-7 lordosis, $\mathrm{C} 0-2$ lordosis, the T1 slope, and thoracic kyphosis. ${ }^{13}$ If the improvement and maintenance of cervical segmental lordosis are better after VBSO, other parameters related to cervical lordosis and sagittal alignment would also improve more. However, because a preserved vertebral body after VBSO provides stable structural support, the maintenance of cervical lordosis may differ between VBSO and ACCF.

The purposes of this study were: 1) to evaluate the improvements in cervical lordosis and sagittal alignment after VBSO through comparison of the postoperative and final evaluation; and 2) to compare the maintenance or changes in cervical lordosis between 1- vs 2-level VBSO and VBSO versus ACCF in patients with cervical spondylotic myelopathy and kyphosis.

\section{Methods \\ Patients}

This retrospective study was approved by the IRB of Asan Medical Center. We reviewed all patients who underwent VBSO between 2007 and 2016. One of the authors (D.H.L.) performed all operations. VBSO was trialed in our institution in 2011, and patients who had undergone VBSO treated after 2012 were recruited to account for the learning curve with this procedure. The inclusion criteria were diagnosis of cervical spondylotic myelopathy and kyphosis, and follow-up for more than 2 years. The exclusion criteria were as follows: 1) diagnosis of a tumor, infection, or fracture; 2) simultaneous posterior cervical fusion; 3) previous cervical spine operation; and 4) thoracolumbar spine surgery.

Thirty-four patients who had undergone VBSO were included. The average age at operation was 52 years (range 27-77 years) and the average follow-up duration was 3 years and 2 months (range 2 years to 4 years 1 month). There were 24 men and 10 women included. Nineteen patients underwent a 2-level operation (Fig. 1). For the comparison with ACCF, patients with the same inclusion and exclusion criteria were recruited. Because simultaneous posterior cervical fusion was performed in all patients who had undergone 2-level ACCF, 31 patients with only 1-level ACCF were included as a control group. The average age in this group was 52 years (range $46-80$ years) and the average follow-up duration was 4 years (range 2 years to 9 years 8 months). There were 19 men and 12 women.

\section{Surgical Procedures \\ VBSO Technique}

For the VBSO technique, ${ }^{5}$ the cervical spine was approached through a standard anterior transverse incision. Initially, a multilevel discectomy was performed at the upper and lower levels of vertebral bodies. The posterior longitudinal ligament at the discectomy level was resected for free anterior translation of the vertebral body. Removing the uncinate process makes the width of the mobile fragment of vertebral bodies wider and decompresses the foramina. Using a 2-3-mm high-speed burr, two parallel longitudinal slits were made at the base of the uncinate process, and the posterior margin of the vertebral body was trimmed to make the vertebral body a box-shaped mobile segment. The Caspar pins should be placed into the vertebral bodies above and below the osteotomy level with distraction at the time of mobilizing vertebral body fragments. As the vertebral bodies were directly pulled out using Allis forceps, successful anterior translation was confirmed by comparing the distances of the protruding anterior portions of the vertebral bodies. The interbody cages were packed with bone fragments harvested from endplate bone spurs and placed into each disc space using an impactor, grasping the mobile fragments using several Allis forceps and pulling them out with a gentle force. The protruding portions of the bodies were partially removed for uniformity and anterior plating was added to enhance stability and to prevent graft extrusion. All patients were requested to use a hard cervical brace for 6 weeks. Routine radiography was obtained postoperatively at day 3,1 month, 6 months, 1 year, and 2 years.

\section{ACCF Technique}

For the ACCF technique, a traditional anterior cervical corpectomy was performed. Ossifications of the posterior longitudinal ligament (OPLLs), hypertrophic posterior longitudinal ligaments, and hypertrophic bone spurs were removed. Following the preparation of adjacent endplates, a titanium mesh cage or allograft was inserted, and anterior plating was added for additional stability.

\section{Variables and Measurements}

The visual analog scale for neck pain (VAS-NP) and arm pain (VAS-AP), Neck Disability Index (NDI), and Japanese Orthopaedic Association (JOA) score were used to assess the degree of disability. Cervical spine lateral radiographs were obtained with the patient in a comfortable upright position, with the upper extremities positioned naturally at the side of the body and with a horizontal gaze parallel to the Frankfort horizontal plane..$^{14}$ Wholespine standing lateral radiographs were obtained with the patient in a standardized position with the arms straight forward and folded.

Radiological data were measured twice by one orthopedic surgeon who did not have any information about the patients (K.B.P.). C0-2 lordosis was measured as the angle 

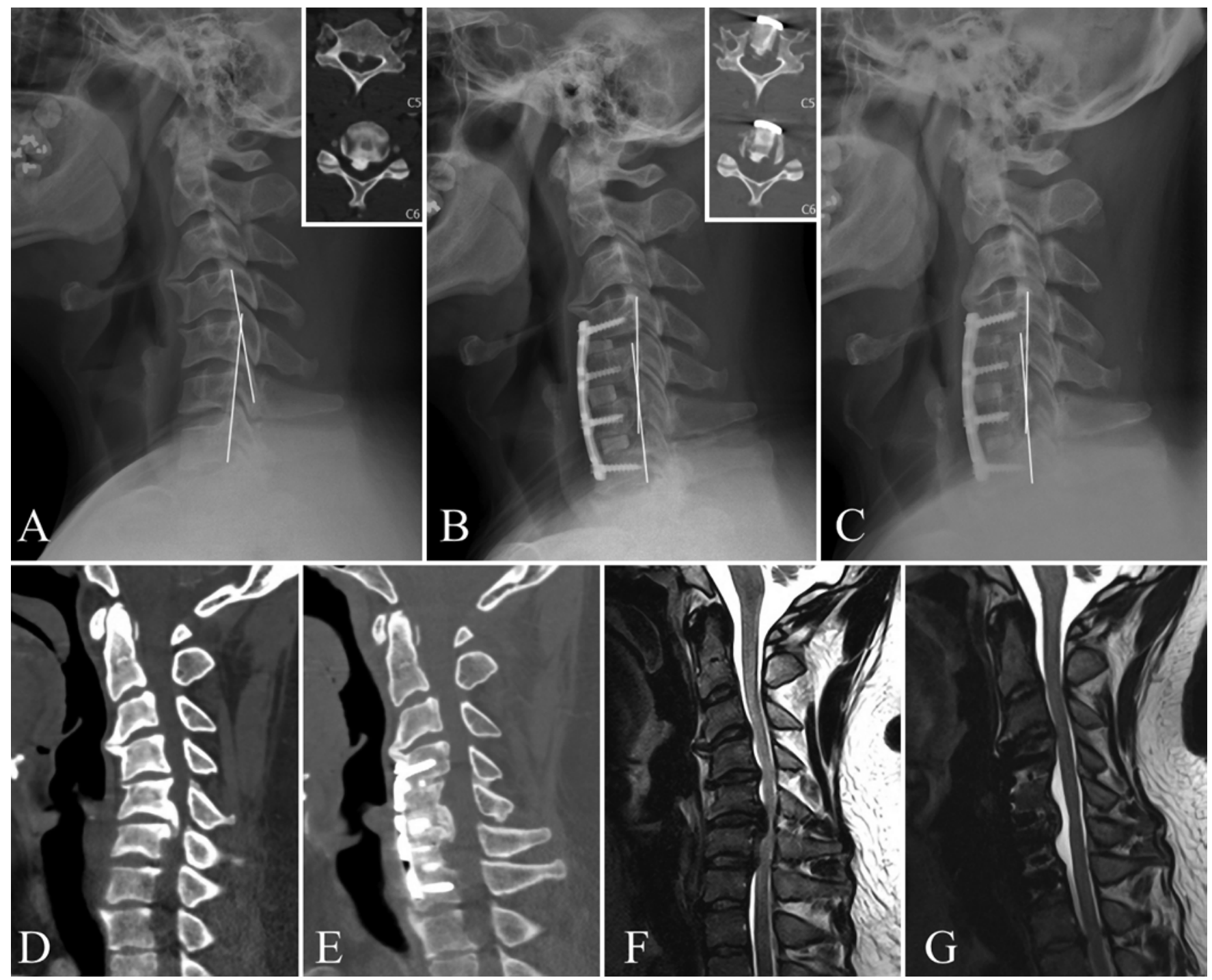

FIG. 1. A 27-year-old man with cervical spondylotic myelopathy due to OPLL and cervical kyphosis underwent VBSO at C5 and C6. On the preoperative radiographic examination (A), the segmental lordosis between $\mathrm{C} 4$ and $\mathrm{C} 7$ was $16^{\circ}$ of kyphosis, and OPLL was noted on CT. After the operation (B), the segmental lordosis between C4 and C7 increased to $4^{\circ}$ of lordosis, and the OPLL in the spinal canal moved outward. At 2 years after the index operation (C), the complete union was noted, and the segmental lordosis between $\mathrm{C} 4$ and $\mathrm{C} 7$ was maintained at $7^{\circ}$. On the sagittal reconstructed CT image, the preoperative narrow spinal canal (D) increased after the operation (E). The preoperative compressed spinal cord on the MR image (F) recovered to the width of the spinal cord, and the space of the dural sac was restored (G).

between the line from the anterosuperior border of the atlas to the inferior end of the occiput and lower endplates of C2. C2-7 lordosis was defined by Cobb's angle between the lower endplates of C2 and C7. Segmental lordosis was defined as the tangential angle between the upper body and lower body of fused segments, because posterior tangential angles reflect the slopes along the curve and can provide an analysis of any buckled areas of the cervical curve. ${ }^{15} \mathrm{C} 2-7$ sagittal vertical axis (SVA) was defined as the distance from the posterosuperior corner of $\mathrm{C} 7$ and a vertical line from the center of the $\mathrm{C} 2$ body (the point of intersection of crossing diagonals of the vertebral body). The T1 slope was measured as the angle between the upper endplate of T1 and a horizontal line. Thoracic kypho- sis was measured using Cobb's angle between the upper endplate of T1 and the lower endplate of T12. Lumbar lordosis was defined by Cobb's angle between both upper endplates of L1 and S1. The sacral slope was evaluated as the angle between the upper endplates of the sacrum and the horizontal line. C7-S1 SVA was defined as the distance from the posterosuperior corner of $\mathrm{S} 1$ and a vertical line from the center of the $\mathrm{C} 7$ body. All plumblines anterior to the vertebral body were positive, and those posterior were negative.

\section{Statistical Analysis}

Statistical analysis was performed using SPSS (version 22.0, IBM Corp.). All continuous variables were tested for 
normality using the Shapiro-Wilk test, which allowed the normal distribution assumption. Paired t-tests were used to compare preoperative, postoperative, and final followup (2 years postoperative) radiological parameters. Independent t-tests were used to compare radiological parameters between 1- and 2-level operation, and 1-level VBSO and 1-level ACCF. The intraclass correlation coefficient (ICC) was used to define intraobserver reliability. Values are expressed as means and standard deviations. The level of significance was set at $\mathrm{p}<0.05$.

\section{Results}

The average operative duration of VBSO was 146.4 minutes (range 100-280 minutes). The osteotomy levels were C4 ( $=1)$, C5 $(n=10)$, C6 $(n=4), C 4-5(n=8)$, and C5-6 $(\mathrm{n}=11)$. The improvement in the VAS-NP was not statistically significant (preoperative $3.3 \pm 2.3$, final follow-up $2.2 \pm 2.1, \mathrm{p}=0.074)$, but the VAS-AP was improved after the operation (preoperative $4.1 \pm 2.7$, final follow-up $2.5 \pm 2.0, \mathrm{p}=0.04)$. The NDI improved from a preoperative score of $14.7 \pm 7.6$ to a final follow-up score of $6.6 \pm 4.8(\mathrm{p}<0.001)$. JOA scores improved at the final follow-up $(15.1 \pm 1.9)$ compared to preoperative values $(12.4 \pm 2.2, \mathrm{p}<0.001)$. Pseudo-motion was noted in $2 \mathrm{pa}-$ tients who had undergone 2-level VBSO. There were no significant neurological complications and union was confirmed at the final follow-up in all VBSO cases. However, in the 1-level ACCF group, the corpectomy levels were C4 $(n=1)$, C5 $(n=22)$, and C6 $(n=8)$, and neurological deficit $(n=2)$, CSF leakage $(n=4)$, graft dislodgement ( $=3)$, hematoma removal $(\mathrm{n}=1)$, and pseudo-motion $(\mathrm{n}=$ 7) were noted (Fig. 2).

The ICCs were 0.927 (T1 slope) to 0.985 (C2-7 lordosis) on the cervical lateral radiographs and 0.950 (pelvic tilt) to 0.984 (C7-S1 SVA) on whole-spine lateral radiographs.

\section{Improvement of Cervical Lordosis and Sagittal Alignment After VBSO}

$\mathrm{C} 0-2$ lordosis gradually decreased, although there was no statistical significance $(\mathrm{p}=0.114$ and $\mathrm{p}=0.629$, respectively), and the final $\mathrm{C} 0-2$ lordosis was significantly improved compared to the preoperative value $(\mathrm{p}=0.049)$. C2-7 lordosis $(\mathrm{p}=0.004)$ and segmental lordosis $(\mathrm{p}<$ 0.001 ) improved postoperatively, and there was no statistical difference between postoperative and final follow-up values ( $p=0.096$ and $p=0.906$, respectively). C2-7 SVA at final follow-up was significantly decreased compared with the preoperative $(\mathrm{p}=0.038)$ and postoperative values $(\mathrm{p}=0.019)$, although there was no significant difference postoperatively $(\mathrm{p}=0.894$; Table 1$)$. In the comparison between preoperative and final follow-up values, not only $\mathrm{C} 2-7$ lordosis and segmental lordosis, but also $\mathrm{C} 0-2$ lordosis and C2-7 SVA, improved after VBSO (Fig. 3). There was not a significant change in $\mathrm{T} 1$ slope, thoracic kyphosis, lumbar lordosis, or C7-S1 SVA.

\section{Comparison of 1- Versus 2-Level VBSO}

There was no difference in preoperative radiographic parameters between the 1- and 2-level VBSO groups (Table 2). The postoperative segmental lordosis was larger in 2-level VBSO, but there was not a significant difference between groups at the final follow-up. In both groups, the improved segmental lordosis $(\mathrm{p}<0.001)$ was not changed until final follow-up in 1-level $(\mathrm{p}=0.062)$ and 2-level $(\mathrm{p}=$ 0.466) VBSO.

\section{Comparison of 1-Level VBSO Versus 1-Level ACCF}

There was no difference in preoperative and postoperative radiographic parameters between the 1-level VBSO and 1-level ACCF groups. However, final segmental lordosis was larger in the VBSO group (Table 3). In both groups, segmental lordosis improved at the final follow-up com-
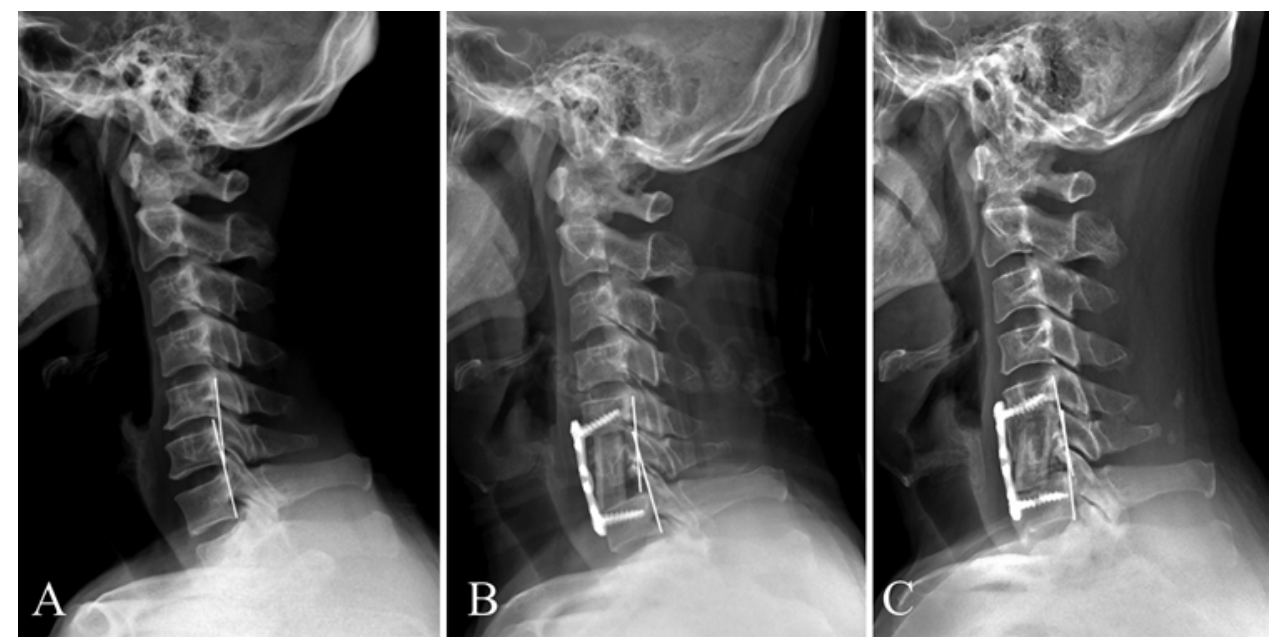

FIG. 2. A 51-year-old woman was treated with ACCF at C6. The preoperative segmental lordosis between $\mathrm{C} 5$ and $\mathrm{C} 7$ was $7^{\circ}(\mathbf{A})$. The immediate postoperative segmental cervical lordosis between C5 and C7 increased up to $14^{\circ}$ (B). At 6 years postoperatively, the segmental lordosis between $\mathrm{C} 5$ and $\mathrm{C} 7$ decreased to $5^{\circ}$ of kyphosis (C). Screw breakage was noted, and the graft showed slight dislodgement, compared with the immediate postoperative radiograph. 
TABLE 1. Comparison of radiological parameters between preoperative, postoperative, and final evaluation

\begin{tabular}{lcccccc}
\hline & & & & \multicolumn{3}{c}{$p$ Value } \\
\cline { 3 - 7 } \multicolumn{1}{c}{ Parameter } & Preop & Postop & Final & $\begin{array}{c}\text { Preop vs } \\
\text { Postop }\end{array}$ & $\begin{array}{c}\text { Postop vs } \\
\text { Final }\end{array}$ & $\begin{array}{c}\text { Preop vs } \\
\text { Final }\end{array}$ \\
\hline C0-2 lordosis $\left(^{\circ}\right)$ & $41.3 \pm 7.1$ & $39.7 \pm 7.0$ & $39.3 \pm 7.2$ & 0.114 & 0.629 & $0.049^{*}$ \\
\hline C2-7 lordosis $\left({ }^{\circ}\right)$ & $7.1 \pm 12.8$ & $11.6 \pm 10.9$ & $13 \pm 9.9$ & $0.004^{*}$ & 0.096 & $<0.001^{*}$ \\
\hline Segmental lordosis $\left(^{\circ}\right)$ & $3.1 \pm 9.2$ & $15 \pm 10.3$ & $15.2 \pm 8.5$ & $<0.001^{*}$ & 0.906 & $<0.001^{*}$ \\
\hline C2-7 SVA $(\mathrm{mm})$ & $21.5 \pm 11.7$ & $21.7 \pm 8.0$ & $18.4 \pm 7.9$ & 0.894 & $0.019^{*}$ & $0.038^{*}$ \\
\hline T1 slope $\left(^{\circ}\right)$ & $24.1 \pm 7.2$ & & $24.6 \pm 6.3$ & & & 0.662 \\
\hline Thoracic kyphosis $\left(^{\circ}\right)$ & $37.7 \pm 12.0$ & & $37.9 \pm 10.9$ & & & 0.837 \\
\hline Lumbar lordosis $\left(^{\circ}\right)$ & $48.4 \pm 13.8$ & & $49.0 \pm 12.5$ & & & 0.663 \\
\hline C7-S1 SVA $(\mathrm{mm})$ & $2.1 \pm 23.8$ & & $11.7 \pm 22.8$ & & & 0.098 \\
\hline
\end{tabular}

${ }^{*}$ Statistically significant difference $(p<0.05)$.

pare to preoperatively ( $\mathrm{p}<0.001$ for $\mathrm{VBSO}, \mathrm{p}=0.012$ for ACCF). However, in the ACCF group, the postoperatively improved segmental lordosis $(\mathrm{p}<0.001)$ decreased at final follow-up $(\mathrm{p}=0.045)$. In contrast, segmental lordosis increased postoperatively $(\mathrm{p}<0.001)$ and was maintained until final follow-up ( $\mathrm{p}=0.062)$ after VBSO (Fig. 4).

\section{Discussion}

Cervical lordosis is needed to maintain forward gazing, and loss of cervical lordosis is associated with pain, functional disability, and decreased vertebral artery hemodynamics because of the stretched vertebral artery course. ${ }^{10,16}$ Furthermore, surgical treatment of cervical myelopathy in patients with less cervical lordosis is difficult and troublesome. Laminoplasty cannot improve symptoms in patients with K-line (-) cervical myelopathy, because of an insufficient posterior shift of the spinal cord. ${ }^{17}$ ACCF is challenging to perform at multiple levels due to risks of significant blood loss and CSF leakage and is occasionally accompanied by complications related to stability. ${ }^{2,4}$ VBSO was developed for safer anterior decom-
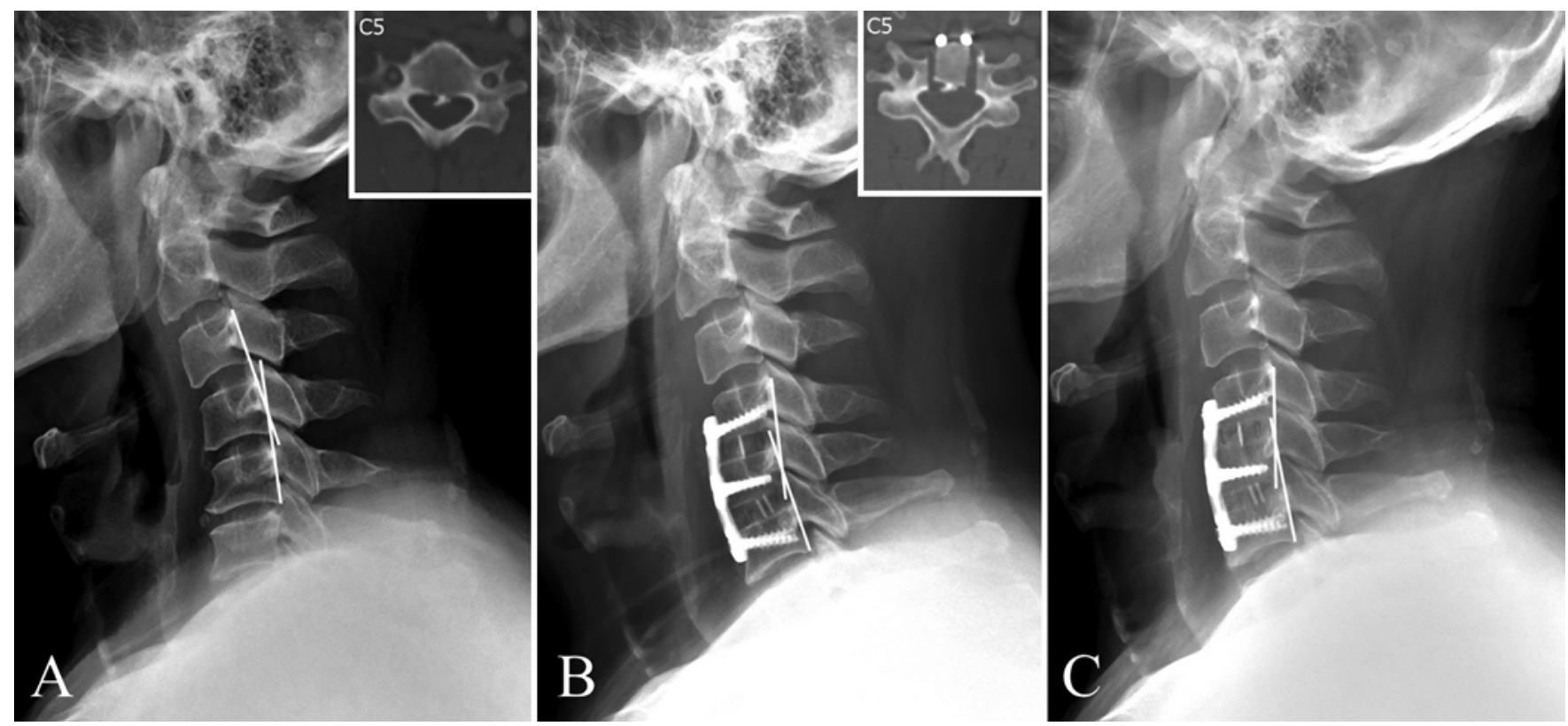

FIG. 3. On radiography, the preoperative cervical alignment (A) of a 48 -year-old man was $57^{\circ}$ of $\mathrm{CO}-2$ lordosis, $-7^{\circ}$ of $\mathrm{C} 2-7$ lordosis, $-9^{\circ}$ of C4 and C6 segmental lordosis, and $28.6 \mathrm{~mm}$ of C2-7 SVA. After VBSO at C5 (B), the cervical lordosis increased, and the postoperative cervical alignment was $50^{\circ}$ of $\mathrm{C} 0-2$ lordosis, $9^{\circ}$ of $\mathrm{C} 2-7$ lordosis, $10^{\circ}$ of $\mathrm{C} 4$ and $\mathrm{C} 6$ segmental lordosis, and $29.9 \mathrm{~mm}$ of C2-7 SVA. OPLL in the spinal canal had moved outward on postoperative CT. At the final follow-up (C), cervical alignment was $46^{\circ}$ of $\mathrm{C} 0-2$ lordosis, $5^{\circ}$ of $\mathrm{C} 2-7$ lordosis, $13^{\circ}$ of $\mathrm{C} 4$ and $\mathrm{C} 6$ segmental lordosis, and $26.1 \mathrm{~mm}$ of $\mathrm{C} 2-7 \mathrm{SVA}$. C0-2 lordosis and C2-7 SVA had decreased, compared with the preoperative values. 
TABLE 2. Comparisons of radiographic measurements between 1- and 2-level VBSO

\begin{tabular}{cccc}
\hline Measurement & 1-Level & 2-Level & p Value \\
\hline Preop & & & \\
\hline C0-2 lordosis $\left({ }^{\circ}\right)$ & $40.6 \pm 2.8$ & $41.8 \pm 9.3$ & 0.601 \\
\hline C2-7 lordosis $\left(^{\circ}\right)$ & $5.2 \pm 10.3$ & $8.5 \pm 14.6$ & 0.462 \\
\hline Segmental lordosis $\left(^{\circ}\right)$ & $0.3 \pm 7.5$ & $5.3 \pm 10.0$ & 0.118 \\
\hline C2-7 SVA $(\mathrm{mm})$ & $21.6 \pm 10.6$ & $21.4 \pm 12.8$ & 0.955 \\
\hline Postop & & & \\
\hline C0-2 lordosis $\left({ }^{\circ}\right)$ & $41.1 \pm 6.2$ & $38.6 \pm 7.6$ & 0.300 \\
\hline C2-7 lordosis $\left(^{\circ}\right)$ & $8.1 \pm 6.2$ & $14.4 \pm 13.0$ & 0.098 \\
\hline Segmental lordosis $\left({ }^{\circ}\right)$ & $10.3 \pm 5.5$ & $18.8 \pm 11.6$ & $0.014^{*}$ \\
\hline C2-7 SVA $(\mathrm{mm})$ & $23.1 \pm 6.4$ & $20.6 \pm 9.1$ & 0.376 \\
\hline Final & & & \\
\hline C0-2 lordosis $\left({ }^{\circ}\right)$ & $39.2 \pm 6.0$ & $39.4 \pm 8.4$ & 0.948 \\
\hline C2-7 lordosis $\left({ }^{\circ}\right)$ & $10.5 \pm 6.2$ & $15.0 \pm 11.8$ & 0.161 \\
\hline Segmental lordosis $\left({ }^{\circ}\right)$ & $12.5 \pm 6.2$ & $17.3 \pm 9.6$ & 0.084 \\
\hline C2-7 SVA $(m m)$ & $18.2 \pm 6.8$ & $18.5 \pm 8.8$ & 0.922 \\
\hline
\end{tabular}

* Statistically significant difference $(p<0.05)$.

pression by anterior translation of the vertebral body. ${ }^{5,6}$ In this study, we evaluated the improvement of cervical lordosis and sagittal alignment after VBSO, because the preserved vertebral body and ACDF above and below the osteotomy may improve cervical lordosis more effectively.

C2-7 and segmental lordosis increased markedly after VBSO. Basques et al. compared multilevel and singlelevel ACDF and observed that multilevel fusions provided considerably greater restoration of lordosis. ${ }^{12}$ During VBSO, the patient undergoes at least 2-level ACDF above and below the VBSO level. The multiple ACDF results in a more significant increase in cervical lordosis, and the postoperative segmental lordosis was larger in 2-level VBSO than in 1-level VBSO. With increasing level of osteotomy, VBSO may increase cervical lordosis to a greater degree compared to ACCF (Fig. 5).

Although segmental lordosis improved after both VBSO and ACCF, segmental lordosis in the ACCF group decreased at the final follow-up, compared with postoperative values. In a previous study comparing multilevel discectomy and corpectomy, 5.1\% subsidence and 2.6\% graft dislodgement were reported in a corpectomy group, whereas there was no case of graft- or instrument-related complication in a discectomy group..$^{18}$ The decrease of segmental lordosis after ACCF in this study may be related to graft subsidence or dislodgement. However, there was no change in the VBSO group, because 4 endplates with the preserved vertebral body provide structural support to prevent graft subsidence, compare to 2 endplates in ACCF. Concerning mechanical support to maintain segmental lordosis, VBSO may be more stable than ACCF.

Villavicencio et al. ${ }^{11}$ reported that maintained or improved segmental sagittal alignment is related to a higher degree of improvement in SF-36 physical component scores, and the severity of disability increases with positive sagittal malalignment after surgery. ${ }^{8,10}$ C2-7 SVA at
TABLE 3. Comparisons of radiographic measurements between the 1-level VBSO and ACCF groups

\begin{tabular}{cccc}
\hline Measurement & VBSO & ACCF & p Value \\
\hline Preop & & & \\
\hline C0-2 lordosis $\left(^{\circ}\right)$ & $40.6 \pm 2.8$ & $39.7 \pm 5.9$ & 0.493 \\
\hline C2-7 lordosis $\left(^{\circ}\right)$ & $5.2 \pm 10.3$ & $4.4 \pm 8.9$ & 0.776 \\
\hline Segmental lordosis $\left(^{\circ}\right)$ & $0.3 \pm 7.5$ & $2.8 \pm 7.2$ & 0.275 \\
\hline C2-7 SVA $(\mathrm{mm})$ & $21.6 \pm 10.6$ & $21.5 \pm 11.1$ & 0.988 \\
\hline Postop & & & \\
\hline C0-2 lordosis $\left(^{\circ}\right)$ & $41.1 \pm 6.2$ & $39.3 \pm 7.6$ & 0.426 \\
\hline C2-7 lordosis $\left(^{\circ}\right)$ & $8.1 \pm 6.2$ & $6.4 \pm 8.1$ & 0.460 \\
\hline Segmental lordosis $\left(^{\circ}\right)$ & $10.3 \pm 5.5$ & $9.3 \pm 7.0$ & 0.639 \\
\hline C2-7 SVA $(\mathrm{mm})$ & $23.1 \pm 6.4$ & $24.0 \pm 10.8$ & 0.759 \\
\hline Final & & & \\
\hline C0-2 lordosis $\left({ }^{\circ}\right)$ & $39.2 \pm 6.0$ & $39.4 \pm 6.2$ & 0.910 \\
\hline C2-7 lordosis $\left({ }^{\circ}\right)$ & $10.5 \pm 6.2$ & $7.9 \pm 7.8$ & 0.268 \\
\hline Segmental lordosis $\left(^{\circ}\right)$ & $12.5 \pm 6.2$ & $7.2 \pm 7.6$ & $0.023^{*}$ \\
\hline C2-7 SVA $(m m)$ & $18.2 \pm 6.8$ & $21.7 \pm 12.0$ & 0.301 \\
\hline
\end{tabular}

* Statistically significant difference $(p<0.05)$.

final follow-up had improved compared to the preoperative value after VBSO. Furthermore, $\mathrm{C} 0-2$ lordosis decreased at final follow-up after VBSO. C0-2 lordosis is related to forward gaze and is known to be negatively correlated with $\mathrm{C} 2-7$ lordosis. If $\mathrm{C} 2-7$ lordosis decreased, a compensation mechanism is activated by increasing $\mathrm{C} 0-2$ lordosis and causing excessive stress on the upper cervical spine due to $\mathrm{C} 0-2$ hyperlordosis. ${ }^{13,19,20} \mathrm{C} 0-2$ hyperlordosis may spontaneously improve after VBSO through better correction of C2-7 lordosis.

Cervical sagittal parameters are related to thoracolumbar sagittal parameters. ${ }^{13,20,21}$ Lee et al. demonstrated that surgical correction of cervical kyphosis affected the T1 slope and indicated that restoration of cervical lordosis increases thoracic kyphosis, but not in the lumbosacral spine. ${ }^{13}$ However, the T1 slope and other thoracolumbosacral parameters did not show any statistical differences in this study. These findings may be related to the normal range of preoperative thoracic kyphosis with wide standard deviations. The increase of T1 slope related to the correction of cervical kyphosis may be associated with thoracic kyphosis; however, this was difficult to demonstrate statistically in this study because of the wide variation in the normal thoracic kyphosis angle.

We could not compare the outcome of multilevel VBSO and ACCF because none of the patients underwent ACCF alone involving more than 2 levels. Patients who underwent only anterior multilevel corpectomy demonstrated a high rate of instability (33\%) in a previous study, so we usually added posterior fusion if a patient needed 2-level corpectomy. ${ }^{2}$ However, pseudo-motion was noted in only $5.9 \%$ of patients (2/34) after 1- and 2-level VBSO, compared with $22.6 \%$ (7/31) after 1-level ACCF. Furthermore, there was no difference in the final cervical lordosis between 1- and 2-level VBSO, and segmental lordosis was larger in 2-level VBSO. VBSO may be a better technique 


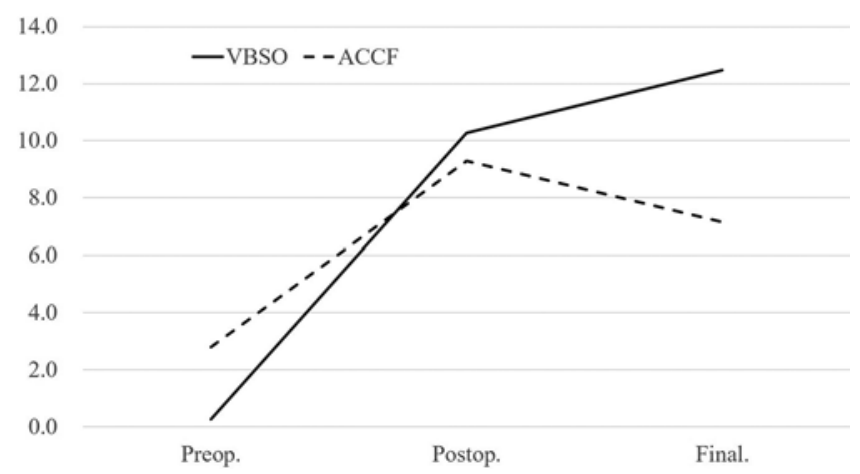

FIG. 4. Changes of segmental lordosis $\left(^{\circ}\right)$ in the VBSO and ACCF groups. In both groups, segmental lordosis improved after the operation; however, this decreased at the final follow-up in the ACCF group.

that can improve cervical lordosis during multilevel anterior decompression without posterior operation. In terms of complications, VBSO was safer than ACCF in this study; however, if the longitudinal slot is too lateral, the vertebral artery may be injured, and if the translation is too much or there is an adhesion, CSF leakage maybe occur. In this situation, a transition to ACCF or a lumbar drain would be helpful.

For patients with multilevel cervical spondylotic my- elopathy, laminoplasty has been recommended, with excellent outcomes. ${ }^{22}$ However, laminoplasty frequently induces progression of OPLL, compared with fusion surgery, and young patients with mixed or continuous types of ossification face a higher risk of progression after laminoplasty ${ }^{23,24}$ Furthermore, postoperative kyphosis after laminoplasty has been reported, and neurological improvement cannot be obtained with posterior decompression alone in patients with K-line (-) cervical myelopathy. ${ }^{17,22,25}$ Another problem is axial pain after posterior cervical spine surgery, which is related to the reconstruction of the extensor musculature and detachment of the semispinalis cervicis muscle. ${ }^{26}$ If patients who have undergone laminoplasty develop OPLL or kyphosis progression, or complain of severe axial pain, VBSO may be the only surgical technique to restore cervical lordosis with multilevel anterior decompression.

This study has several limitations. First, as this was a retrospective study, we could not control the operated vertebral level. Further study about the comparison between 2-level VBSO and ACCF with the same level would be helpful in understanding the amount of improvement and maintenance of cervical lordosis. However, improvement of segmental lordosis was better after 2-level VBSO compared to 1-level VBSO, and the preserved vertebral body may be more useful to maintain the improved cervical lordosis in a 2-level operation. There were no definite ap-

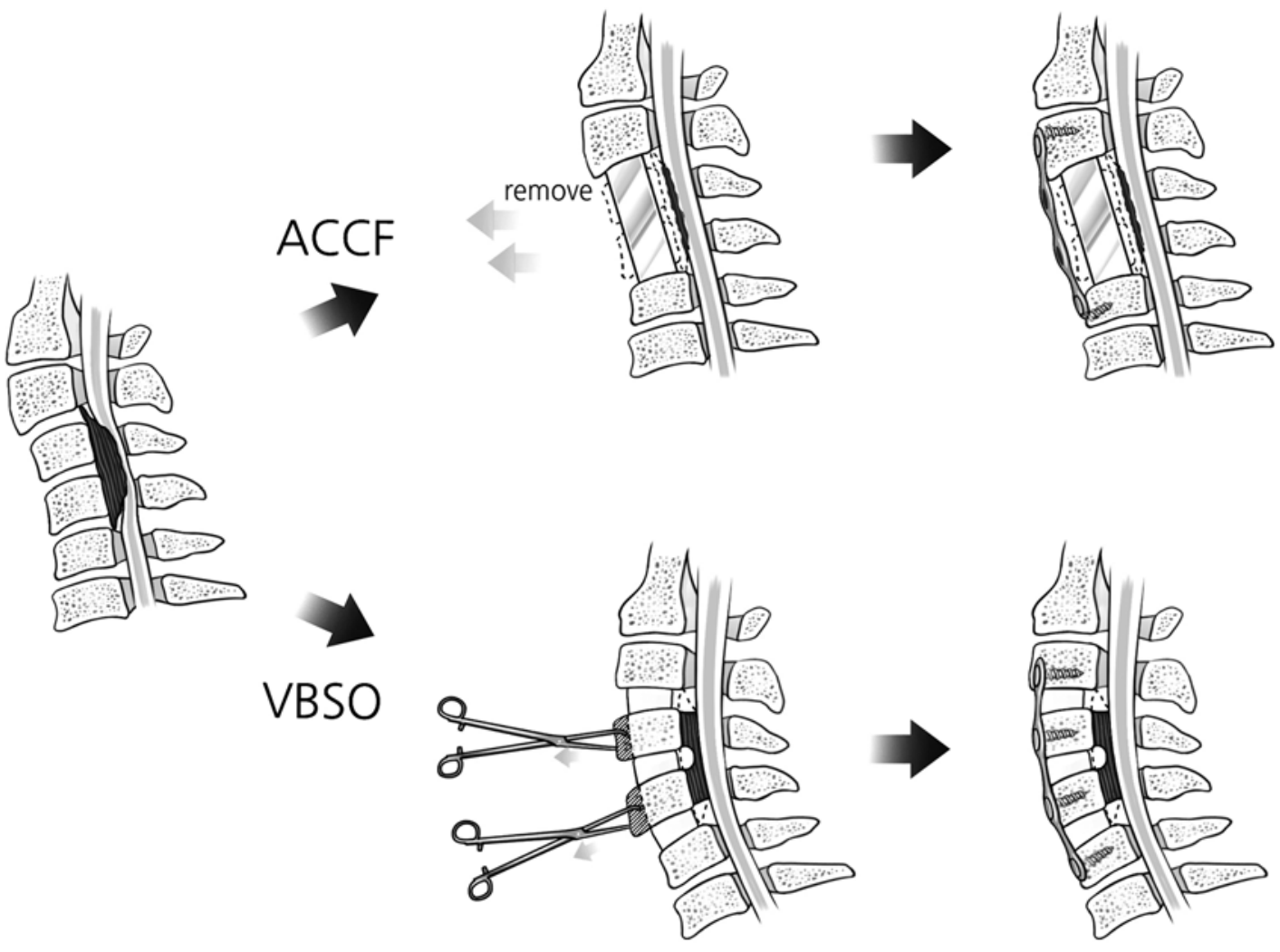

FIG. 5. During ACCF, the height of the vertebral body is the primary concern, and there may be little change in cervical lordosis. However, the height of the vertebral body is maintained during VBSO, because the body merely slides anteriorly, and cervical lordosis increases due to cervical discectomy and fusion with a trapezoidal wedge cage above and below the VBSO level. 
propriate values for cervical lordosis after fusion, although several studies reported $22.4^{\circ}$ for $\mathrm{C} 0-2$ lordosis and $9.9^{\circ}$ for $\mathrm{C} 2-7$ lordosis in asymptomatic adults. ${ }^{21,27}$ The cause of cervical myelopathy is usually located in the anterior column, such as the anterior osteophyte, disc herniation, or OPLL. ${ }^{24}$ In our opinion, more cervical lordosis after fusion would be better in terms of anterior decompression.

\section{Conclusions}

VBSO improves the cervical lordosis in patients with cervical spondylotic myelopathy and kyphosis because multiple ACDFs above and below the VBSO level increased the cervical lordosis more effectively, and because a preserved vertebral body in VBSO provides structural support to prevent graft subsidence. Not only C2-7 lordosis and segmental lordosis, but also $\mathrm{C} 0-2$ lordosis and C2-7 SVA, were improved after VBSO at the final followup. For patients who require correction of kyphosis and multilevel anterior decompression without posterior operation, VBSO may be a reliable technique.

\section{References}

1. Sevki K, Mehmet T, Ufuk T, et al. Results of surgical treatment for degenerative cervical myelopathy: anterior cervical corpectomy and stabilization. Spine (Phila Pa 1976). 2004;29(22):2493-2500.

2. Bayerl SH, Pöhlmann F, Finger T, et al. Two-level cervical corpectomy-long-term follow-up reveals the high rate of material failure in patients, who received an anterior approach only. Neurosurg Rev. 2019;42(2):511-518.

3. Belanger TA, Roh JS, Hanks SE, et al. Ossification of the posterior longitudinal ligament. Results of anterior cervical decompression and arthrodesis in sixty-one North American patients. J Bone Joint Surg Am. 2005;87(3):610-615.

4. Xiao SW, Jiang H, Yang LJ, Xiao ZM. Anterior cervical discectomy versus corpectomy for multilevel cervical spondylotic myelopathy: a meta-analysis. Eur Spine J. 2015;24(1):31-39.

5. Lee DH, Cho JH, Lee CS, et al. A novel anterior decompression technique (vertebral body sliding osteotomy) for ossification of posterior longitudinal ligament of the cervical spine. Spine J. 2018;18(6):1099-1105.

6. Lee DH, Riew KD, Choi SH, et al. Safety and efficacy of a novel anterior decompression technique for ossification of posterior longitudinal ligament of the cervical spine. J Am Acad Orthop Surg. 2019;00:1-10.

7. Faldini C, Pagkrati S, Leonetti D, et al. Sagittal segmental alignment as predictor of adjacent-level degeneration after a cloward procedure. Clin Orthop Relat Res. 2011;469(3):674681.

8. Hyun SJ, Kim KJ, Jahng TA, Kim HJ. Relationship between T1 slope and cervical alignment following multilevel posterior cervical fusion surgery: impact of T1 slope minus cervical lordosis. Spine (Phila Pa 1976). 2016;41(7):E396E402.

9. Park MS, Kelly MP, Lee DH, et al. Sagittal alignment as a predictor of clinical adjacent segment pathology requiring surgery after anterior cervical arthrodesis. Spine J. 2014;14(7):1228-1234.

10. Tang JA, Scheer JK, Smith JS, et al. The impact of standing regional cervical sagittal alignment on outcomes in posterior cervical fusion surgery. Neurosurgery. 2015;76(suppl 1):S14S21.

11. Villavicencio AT, Babuska JM, Ashton A, et al. Prospective, randomized, double-blind clinical study evaluating the cor- relation of clinical outcomes and cervical sagittal alignment. Neurosurgery. 2011;68(5):1309-1316.

12. Basques BA, Louie PK, Mormol J, et al. Multi- versus singlelevel anterior cervical discectomy and fusion: comparing sagittal alignment, early adjacent segment degeneration, and clinical outcomes. Eur Spine J. 2018;27(11):2745-2753.

13. Lee DH, Ha JK, Chung JH, et al. A retrospective study to reveal the effect of surgical correction of cervical kyphosis on thoraco-lumbo-pelvic sagittal alignment. Eur Spine J. 2016;25(7):2286-2293.

14. Lundström A, Lundström F. The Frankfort horizontal as a basis for cephalometric analysis. Am J Orthod Dentofacial Orthop. 1995;107(5):537-540.

15. Harrison DE, Harrison DD, Cailliet R, et al. Cobb method or Harrison posterior tangent method: which to choose for lateral cervical radiographic analysis. Spine (Phila Pa 1976). 2000;25(16):2072-2078.

16. Bulut MD, Alpayci M, Şenköy E, et al. Decreased vertebral artery hemodynamics in patients with loss of cervical lordosis. Med Sci Monit. 2016;22:495-500.

17. Fujiyoshi T, Yamazaki M, Kawabe J, et al. A new concept for making decisions regarding the surgical approach for cervical ossification of the posterior longitudinal ligament: the K-line. Spine (Phila Pa 1976). 2008;33(26):E990-E993.

18. Li Z, Huang J, Zhang Z, et al. A comparison of multilevel anterior cervical discectomy and corpectomy in patients with 4-level cervical spondylotic myelopathy: a minimum 2-year follow-up study. Clin Spine Surg. 2017;30(5):E540-E546.

19. Lafage R, Challier V, Liabaud B, et al. Natural head posture in the setting of sagittal spinal deformity: Validation of chinbrow vertical angle, slope of line of sight, and McGregor's slope with health-related quality of life. Neurosurgery. 2016;79(1):108-115.

20. Lee SH, Kim KT, Seo EM, et al. The influence of thoracic inlet alignment on the craniocervical sagittal balance in asymptomatic adults. J Spinal Disord Tech. 2012;25(2):E41-E47.

21. Lee SH, Son ES, Seo EM, et al. Factors determining cervical spine sagittal balance in asymptomatic adults: correlation with spinopelvic balance and thoracic inlet alignment. Spine J. 2015;15(4):705-712.

22. Kimura I, Shingu H, Nasu Y. Long-term follow-up of cervical spondylotic myelopathy treated by canal-expansive laminoplasty. J Bone Joint Surg Br. 1995;77(6):956-961.

23. Kawaguchi Y, Kanamori M, Ishihara H, et al. Progression of ossification of the posterior longitudinal ligament following en bloc cervical laminoplasty. J Bone Joint Surg Am. 2001;83(12):1798-1802.

24. Lee $\mathrm{CH}$, Sohn MJ, Lee CH, et al. Are there differences in the progression of ossification of the posterior longitudinal ligament following laminoplasty versus fusion?: a metaanalysis. Spine (Phila Pa 1976). 2017;42(12):887-894.

25. Sakai K, Yoshii T, Hirai T, et al. Cervical sagittal imbalance is a predictor of kyphotic deformity after laminoplasty in cervical spondylotic myelopathy patients without preoperative kyphotic alignment. Spine (Phila Pa 1976). 2016;41(4):299-305.

26. Wang S-J, Jiang S-D, Jiang L-S, Dai L-Y. Axial pain after posterior cervical spine surgery: a systematic review. Eur Spine J. 2011;20(2):185-194.

27. Hardacker JW, Shuford RF, Capicotto PN, Pryor PW. Radiographic standing cervical segmental alignment in adult volunteers without neck symptoms. Spine (Phila Pa 1976). 1997;22(13):1472-1480.

\section{Disclosures}

The authors report no conflict of interest concerning the materials or methods used in this study or the findings specified in this paper. 


\section{Author Contributions}

Conception and design: KB Park, DH Lee. Acquisition of data: Cho, JW Park. Analysis and interpretation of data: Cho, JW Park. Drafting the article: KB Park. Critically revising the article: KB Park, DH Lee, Hwang. Reviewed submitted version of manuscript: Hwang. Approved the final version of the manuscript on behalf of all authors: KB Park. Statistical analysis: KB Park.

Administrative/technical/material support: CS Lee. Study supervision: DH Lee, CS Lee.

\section{Supplemental Information}

\section{Previous Presentations}

Portions of this work were presented in abstract form at the
Cervical Spine Research Society 47th Annual Meeting, in New York, New York, on November 22, 2019.

\section{Correspondence}

Kun-Bo Park: Severance Children's Hospital, Yonsei University College of Medicine, Seoul, Korea. pedoskbp@yuhs.ac; kunbopark@gmail.com. 